Research Paper

\title{
Load-balanced routing with selective even traffic splitting*
}

\author{
Masashi HONMA ${ }^{1}$, Shunichi TSUNODA ${ }^{2}$, and Eiji OKI ${ }^{3}$ \\ 1,2,3 The University of Electro-Communications
}

\begin{abstract}
This paper proposes an even-split Smart-OSPF (S-OSPF) scheme to reduce network congestion more than the conventional non-split S-OSPF and to distribute traffic more easily than the conventional split S-OSPF. In split S-OSPF, source edge nodes distribute traffic unevenly to their neighbor nodes, but the implementation becomes involved to split traffic with different distribution. In non-split S-OSPF, source edge nodes transmit traffic to only one neighbor so that network congestion can be minimized, where non-split S-OSPF distributes traffic more simply than split S-OSPF. In the proposed scheme, source edge nodes transmit traffic evenly to selected neighbor nodes to minimize network congestion. The optimization problem to select a suitable set of neighbor nodes for even traffic distribution raised by the proposed scheme is formulated as an Integer Linear Programming (ILP) problem. The difficulty of solving the ILP problem in a practical time leads us to introduce a heuristic algorithm. The performances of our developed heuristic algorithm are evaluated via simulation developed in terms of network size. Numerical results show that even-split S-OSPF offers better routing performance than non-split S-OSPF for small-size networks and matches that of split S-OSPF for large-size networks.
\end{abstract}

\section{KEYWORDS}

IP routing, load balancing, OSPF, optimization problem, heuristic algorithm

\section{Introduction}

An appropriate routing scheme can increase the network resource utilization rate and network throughput of Internet Protocol (IP) networks. Since it optimizes the assignment of traffic resources, additional traffic can be supported. One useful approach to enhancing routing performance is to minimize the maximum link utilization rate, also called the network congestion ratio, of all network links. Reduction of the network congestion ratio leads to an increase in admissible traffic.

Several routing strategies have been extensively studied [1], [3]. Wang et al. [1] formulates a general traffic

Received October 26, 2012; Revised December 25, 2012; Accepted January 8, 2013.

* This work was supported in part by the Ministry of Education, Science, Sports and Culture, Grant-in-Aid for Scientific Research (C) 23500081, and the Support Center for Advanced Telecommunications Technology Research (SCAT). Parts of this paper werepartially presented at the 18th Asia-Pacific Conference on Communications (APCC 2012).

DOI: $10.2201 / \mathrm{NiiPi} .2013 .10 .11$ engineering problem based on the Multi-Protocol Label Switching (MPLS) Traffic-Engineering (TE) technology, where traffic demands are assumed to be flexibly split among source and destination nodes. However, legacy networks mainly employ shortest-pathbased routing protocols such as Open Shortest Path First (OSPF) and Intermediate System to Intermediate System (IS-IS). This means that already deployed IP routers in the legacy networks would need to be upgraded, which would significantly increase capital expenditures. Therefore, routing control which set optimum link weights in OSPF-based networks has been studied.

In OSPF-based networks, all packets are forwarded in shortest path with respect link weights. Therefore, by setting up optimal link costs, routing strategies are controlled. When traffic demands are changed, optimum weights are recalculated and network operators recon- 
figure the IP routes. However, changing frequently link weights causes network instability, which leads to packet loss and the formation of loops.

Mishra et al. presented Smart OSPF (S-OSPF) in [4] in order to improve the performance of legacy OSPFbased networks without changing link weights. In this approach only source edge nodes distribute traffic to the neighbor nodes with optimum ratios that are obtained by solving the Linear Programming (LP) problem. After the traffic reaches the neighbor nodes, it is routed according the OSPF protocol. In S-OSPF, the source node distributes IP packets only to the neighbor nodes. Hence, the implementation of S-OSPF is easier than MPLS-TE in legacy networks running on OSPF, because MPLS-TE needs significant functional upgrades both in hardware and software of routers employing original OSPF.

In S-OSPF presented in [4], [5], each source edge node needs an additional table with optimal distribution ratios for traffic entering into the network. A classical IP forwarding table is used for the transit traffic. A source node unevenly distributes traffic to the neighbor nodes using optimized different distribution ratios. In general, as traffic is split at each source node, we call this scheme split S-OSPF.

From the viewpoint of implementation, split S-OSPF has some problems. First, the source nodes of split S-OSPF distribute traffic unevenly to their neighbor nodes, but most existing routers are unable to split traffic with different distribution ratios into multiple routes. Second, legacy router software and hardware must be improved and updated to implement the uneven traffic distribution function, which would incur large capital expenditures. This problem can be overcome if the source nodes choose only one neighbor for traffic distribution.

There is a scheme to overcome this problem that the source nodes choose only one neighbor node and forward traffic to minimize network congestion. This version of S-OSPF is called non-split S-OSPF to differentiate it from split S-OSPF. This scheme does not distribute traffic. Thus, network congestion is higher than split S-OSPF. However, forward processing is easier than split S-OSPF by not forwarding with different distribution ratio. As network size increases, the difference between split S-OSPF and non-split S-OSPF shrinks. Thus, non-split S-OSPF effectively matched the load-balancing performance of split-S-OSPF for large networks [6]. However, the difference of congestion ratio between split S-OSPF and non-split S-OSPF is large for small-size networks.

A question arises: is it possible to improve the performance of non-split S-OSPF by distributing traffic evenly to the neighbor nodes in the framework of S-
OSPF in networks for which non-splits S-OSPF does not work effectively?

This paper answers to this question. This paper proposes a routing scheme which reduces network congestion ratio more than non-split S-OSPF and distributes traffic easier than split S-OSPF. This version of S-OSPF is called even-split $\mathrm{S}-\mathrm{OSPF}$. Even-split S-OSPF selects a set of suitable neighbor nodes in all neighbor nodes, and forwards traffic evenly to those nodes. Forwarding evenly can reduce network congestion ratio more than non-split S-OSPF and distribute traffic more easily than split S-OSPF. The routing decision problem is formulated as an integer linear programming (ILP) problem. Since it is difficult to solve the ILP problem in practical time, this paper develops a heuristic algorithm.

Figures 1-4 schematically compare, classical Shortest Path Routing (SPR), split S-OSPF, non-split SOSPF, and even-split S-OSPF. Figure 1 shows classical SPR. Traffic between two source-destination pairs is transferred over OSPF shortest paths. Network congestion may occur, while some links remain unused. In classical SPR, traffic is not effectively balanced, and instead becomes concentrated. The split S-OSPF scenario, shown in Figure 2, makes use of network re-

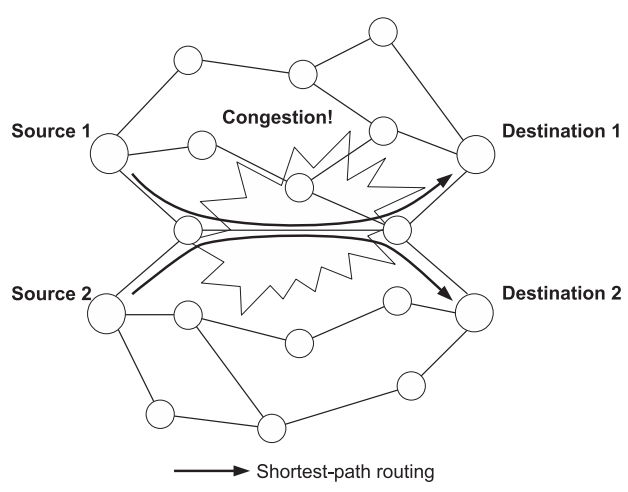

Fig. 1 Classical SPR.

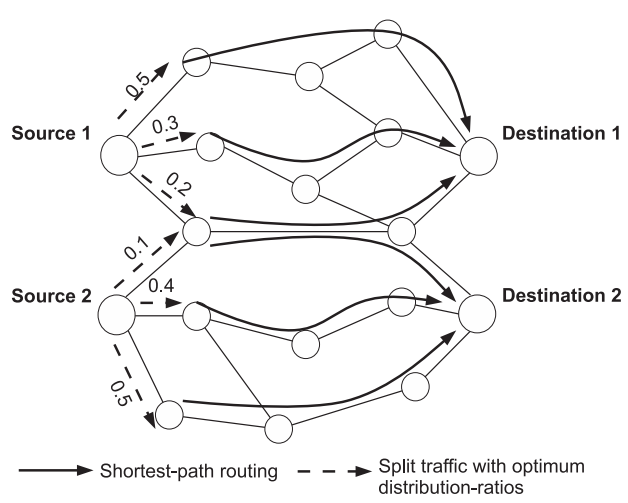

Fig. 2 Split S-OSPF. 


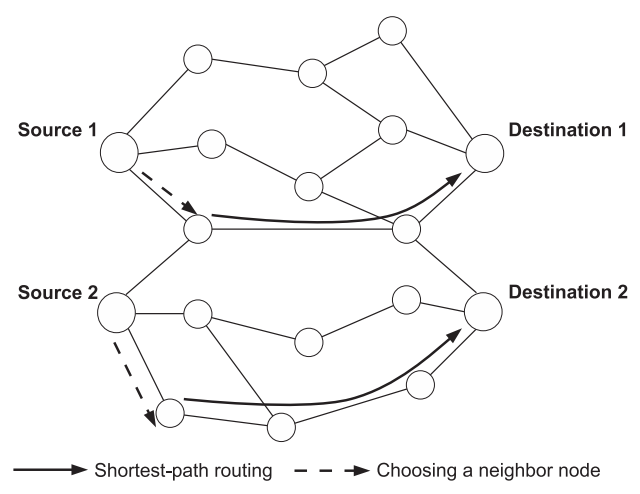

Fig. 3 Non-split S-OSPF.

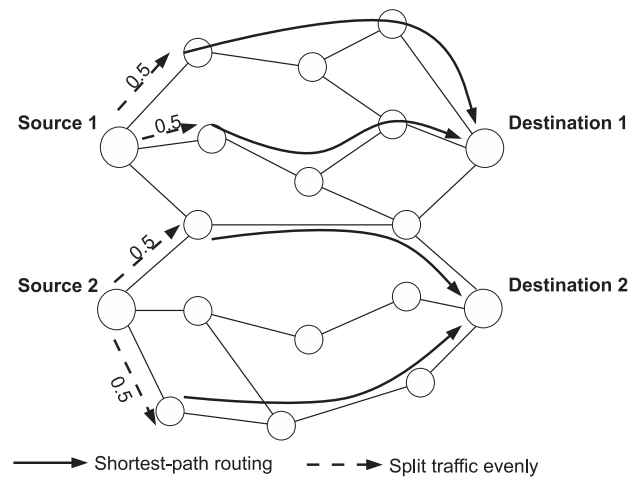

Fig. 4 Even-split S-OSPF.

sources better than classical SPR. In split S-OSPF, since a source node distributes traffic to multiple neighbors, it provides better load balancing. In non-split S-OSPF, as shown in Figure 3, a source node is allowed to transmit traffic only to one neighbor, which is selected so that the maximum link utilization is minimized. Figure 4 shows even-split S-OSPF. In even-split S-OSPF, a source node forwards traffic evenly to a set of suitable neighbor nodes evenly in order to minimize network congestion.

The remainder of this paper is organized as follows. Section 2 uses a network model to introduce the terminology of this paper. Section 3 presents the split S-OSPF scheme. Section 4 presents the non-split SOSPF scheme. Section 5 presents the even-split SOSPF scheme. Section 6 evaluates the performance of the even-split S-OSPF scheme in a comparison against non-split, split S-OSPF and classical SPR schemes. Finally, section 7 summarizes the key points.

\section{Network model}

The network is represented as a directed graph $G(V, E)$, where $V$ is the set of vertices (nodes) and $E$ is the set of links. $Q$ is a set of edge nodes which receive
Table 1 Routing table of Classical OSPF.

\begin{tabular}{c|c}
\hline Prefix & Next hop \\
\hline $130.60 .225 .0 / 24$ & $\mathrm{~A}$ \\
\hline $148.32 .0 .0 / 16$ & $\mathrm{~B}$ \\
\hline $148.32 .96 .0 / 24$ & $\mathrm{C}$ \\
\hline$\vdots$ & $\vdots$ \\
\hline
\end{tabular}

Table 2 Routing table of Split S-OSPF.

\begin{tabular}{|c|c|c|c|c|}
\hline \multicolumn{3}{|c|}{ For traffic entering network } & \multicolumn{2}{|c|}{ For transit traffic } \\
\hline Prefix & Next hop & Distribution ratio & Prefix & Next hop \\
\hline \multirow[t]{3}{*}{$130.60 .225 .0 / 24$} & A & 0.5 & $130.60 .225 .0 / 24$ & A \\
\hline & $\bar{B}$ & 0.2 & $148.32 .0 .0 / 16$ & $\mathrm{~B}$ \\
\hline & $\overline{\mathrm{C}}$ & 0.3 & $148.32 .96 .0 / 24$ & $\mathrm{C}$ \\
\hline \multirow[t]{2}{*}{$148.32 .0 .0 / 16$} & $\mathrm{~B}$ & 0.8 & & \\
\hline & $\mathrm{C}$ & 0.2 & : & : \\
\hline \multirow[t]{3}{*}{$148.32 .96 .0 / 24$} & A & 0.3 & & \\
\hline & $\mathrm{B}$ & 0.3 & & \\
\hline & $\mathrm{C}$ & 0.4 & & \\
\hline$\vdots$ & $\vdots$ & $\vdots$ & & \\
\hline
\end{tabular}

traffic incoming to the network and transmit traffic outgoing from the network, where $Q \subseteq V$. A link from node $i \in V$ to node $j \in V$ is denoted as $(i, j) \in E(i \neq j)$. $x_{i j}^{p q}$ is the portion of the traffic from node $p \in Q$ to node $q \in Q$ routed through $(i, j) \in E . X$ is represented as a four-dimensional routing matrix whose element is $x_{i j}^{p q}$, or $X=\left\{x_{i j}^{p q}\right\} .\{X\}$ is a set of $\mathrm{X} . c_{i j}$ is the capacity of $(i, j) \in E$. The traffic volume passing through $(i, j)$ is donated as $t_{i j} . T=\left\{d_{p q}\right\}$ is the traffic matrix, where $d_{p q}$ is the traffic demand from node $p$ to node $q$. The network congestion ratio, which refers to the maximum value of all link utilization rates in the network, is denoted as $r$, where

$$
r=\max _{(i, j) \in E}\left\{\frac{t_{i j}}{c_{i j}}\right\} .
$$

Minimizing $r$ means that admissible traffic is maximized. The admissible traffic volume is accepted up to the current traffic volume multiplied by $1 / r$. Minimizing $r$ with routing control is the objective of this paper.

To implement S-OSPF, the IP forwarding table at the source node must be changed, unlike classical OSPF. The IP forwarding table that is created by classical OSPF is modified so that traffic can be distributed to the neighbor nodes with the computed distribution ratios. Table 1-4 show examples of IP forwarding tables for classical OSPF, split S-OSPF, non-split S-OSPF and even-split S-OSPF. S-OSPF has two types of IP forwarding tables. One is used for traffic entering the network at the node, where traffic is distibuted to the neighbor nodes. The other, which is the same as that of classical OSPF, is used to handle transit traffic at the 
Table 3 Routing table of Not-split S-OSPF.

\begin{tabular}{|c|c|c|c|}
\hline \multicolumn{2}{|c|}{ For traffic entering network } & \multicolumn{2}{|c|}{ For transit traffic } \\
\hline Prefix & Next hop & Prefix & Next hop \\
\hline $130.60 .225 .0 / 24$ & $\mathrm{C}$ & $130.60 .225 .0 / 24$ & A \\
\hline 148.32.0.0/16 & A & $148.32 .0 .0 / 16$ & B \\
\hline $148.32 .96 .0 / 24$ & B & $148.32 .96 .0 / 24$ & $\mathrm{C}$ \\
\hline$\vdots$ & $\vdots$ & $\vdots$ & $\vdots$ \\
\hline
\end{tabular}

Table 4 Routing table of Even-split S-OSPF.

\begin{tabular}{|c|c|c|c|}
\hline \multicolumn{2}{|c|}{ For traffic entering network } & \multicolumn{2}{|c|}{ For transit traffic } \\
\hline Prefix & Next hop & Prefix & Next hop \\
\hline \multirow[t]{2}{*}{$130.60 .225 .0 / 24$} & A & $130.60 .225 .0 / 24$ & A \\
\hline & B & $148.32 .0 .0 / 16$ & B \\
\hline \multirow[t]{3}{*}{$148.32 .0 .0 / 16$} & A & $148.32 .96 .0 / 24$ & $\mathrm{C}$ \\
\hline & B & : & : \\
\hline & $\mathrm{C}$ & $\cdot$ & \\
\hline \multirow[t]{2}{*}{$148.32 .96 .0 / 24$} & B & & \\
\hline & $\mathrm{C}$ & & \\
\hline. & $\vdots$ & & \\
\hline
\end{tabular}

node.

Split S-OSPF distributes traffic to the neighbor nodes with optimum ratios. Non-split S-OSPF forwords traffic to minimmize network congestion. Even-split SOSPF forwords traffic evenly to selected nodes.

Splitting flow at the source node to the neighbor nodes does not degrade the upper-layer performance measures such as TCP in a backbone IP network. We assume a high-capacity IP backbone network. As each traffic demand, $d_{p q}$, between source edge node $p$ and destination edge node $q$ in the backbone network has large traffic volume, flow splitting can be performed by using end-to-end source and destination IP addresses in the IP packets. The TCP flows that have the same destination addresses are not split.

\section{Split S-OSPF}

In S-OSPF, only source edge nodes distribute traffic to the neighbor nodes with optimum ratio. After the traffic reaches the neighbor nodes, it is routed according the OSPF protocol. Let $O S P F_{\text {nexthop }}{ }^{p q}$ be the next hop node from node $i$ on the shortest path route from node $p$ to node $q$, and $O S P F_{\text {ancestor }} r^{p q}$ be any of the upstream node(s) on the shortest path route from node $p$ to node $q$.

The optimum ratios for traffic distribution to minimize the worst-case network congestion ratio are obtained by solving the following Linear Programming
(LP) problem.

$$
\begin{aligned}
& \min _{\text {s.t. }} x_{i j}^{p q}-\sum_{j r:(j,, i) \in E} x_{j \prime i}^{p q}=0 \\
& p, q \in Q, i \neq p, i \neq q, j=O S P F_{n e x t h o p_{i}^{p q}} \\
& \quad \sum_{j:(i, j) \in E, j \neq O S P F_{\text {ancesto } r_{i}^{p q}}} x_{i j}^{p q}-\sum_{j r:(j, r, i) \in E} x_{j, i}^{p q}=1 \\
& p, q \in Q, i=p \\
& \sum_{p, q \in Q} d_{p q} x_{i j}^{p q} \leq c_{i j} \cdot r \quad(i, j) \in E \\
& 0 \leq x_{i j}^{p q} \leq 1 \quad p, q \in Q,(i, j) \in E \\
& 0 \leq r \leq 1
\end{aligned}
$$

The objective function in Eqs. (2a) says that the variable to be minimized is the maximum link utilization across all the links. Eqs. (2b) and (2c) specify the flow conservation constraints. Eq. (2b) represents the fact that the traffic flowing into a node must be equal to the traffic flowing out of the node for any node other than the source node and the destination node for each demand. Eq. (2c) says that the net flow out of the source node is 1 , which is the total required normalized bandwidth of the traffic demand. Eq. (2d) is the link capacity utilization constraint. It makes sure that the sum of the fractions of traffic demands routed over a link should not exceed the maximum link utilization times the total capacity of the link.

\section{Non-split S-OSPF}

An optimal routing formulation for non-split SOSPF that yields distribution ratio $x_{i j}^{p q}$ is as follows.

$$
\begin{aligned}
& \min _{\text {s.t. }} x_{i j}^{p q}-\sum_{j r:(j,, i) \in E} x_{j ; i}^{p q}=0 \\
& p, q \in Q, i \neq p, i \neq q, j=O S P F_{n e x t h o p_{i}^{p q}} \\
& \sum_{j:(i, j) \in E, j \neq O S P F_{\text {ancessor } r_{i}^{p q}}} x_{i j}^{p q}-\sum_{j r:(j, i) \in E} x_{j r i}^{p q}=1 \\
& p, q \in Q, i=p \\
& \sum_{p, q \in Q} d_{p q} x_{i j}^{p q} \leq c_{i j} \cdot r \quad(i, j) \in E \\
& x_{i j}^{p q}=\{0,1\} \quad p, q \in Q,(i, j) \in E \\
& 0 \leq r \leq 1
\end{aligned}
$$

Eq. (3e) specifies the constraint that $x_{i j}^{p q}$ is limited to values of 0 or 1 . The formulation for the non-split case 
presented in Eqs. (3a)-(3f) is an Integer Liner Programming (ILP) problem, which is NP-hard. Therefore, developing a heuristic algorithm is required.

\section{Even split S-OSPF}

\subsection{ILP formulation}

In even-split S-OSPF, only source edge nodes distribute traffic evenly to the neighbor nodes. $m_{p q}$ is the number of equal split from a source node to the neighbor nodes. $y_{i j}^{p q}$ is the portion of integer, where

$$
y_{i j}^{p q}=x_{i j}^{p q} \cdot m_{p q} .
$$

An optimal routing formulation for even-split SOSPF that yields distribution ratio $y_{i j}^{p q}$ is as follows.

$$
\begin{aligned}
& \min _{\text {s.t. }} y_{i j}^{p q}-\sum_{j r:(j,, i) \in E} y_{j r i}^{p q}=0 \\
& p, q \in Q, i \neq p, i \neq q, j=O S P F_{\text {nexthop }}^{p q} \\
& \sum_{j:(i, j) \in E, j \neq O S P F_{\text {ancestop }}^{p q}} y_{i j}^{p q}-\sum_{j r:(j,, i) \in E} y_{j, i}^{p q}=m_{p q} \\
& p, q \in Q, i=p \\
& \sum_{p, q \in Q} d_{p q} y_{i j}^{p q} \leq c_{i j} \cdot r \cdot m_{p q} \quad(i, j) \in E \\
& 0 \leq y_{i j}^{p q} \leq 1(i=p), \quad p, q \in Q,(i, j) \in E \\
& 0 \leq y_{i j}^{p q} \leq m_{p q}(i \neq p), \quad p, q \in Q,(i, j) \in E \\
& 0 \leq r \leq 1
\end{aligned}
$$

Eqs. (5b) and (5c) specify the flow conservation constraints. The formulation for the even-split case presented in Eqs. (5a)-(5g) are an Integer Liner Programming (ILP) problem, which is NP-hard. Therefore, developing a heuristic algorithm is required.

\subsection{Heuristic algorithm}

We introduce a heuristic algorithm against the ILP problem. In the algorithm, first, the corresponding LP problem is solved by assuming $x_{i j}^{p q}$ is a real number, where $0 \leq x_{i j}^{p q} \leq 1$. Based on the LP solution, if any node pair $(p, q)$ whose traffic demands are not split exists, the corresponding $x_{i j}^{p q}$ is considered as a part of the solution to the ILP problem. Second, for other node pairs, by selecting each $(p, q)$ one by one, the corresponding traffic demands are allocated in a sequential manner which makes the congestion ratio as small as possible.

$\Phi$ and $\Psi$ are defined prior describing the algorithm as follows. $\Phi$ is defined as a set of source and destination node pairs whose traffic demands are not split. $\Psi$ is defined as a set of source and destination node pairs whose traffic demands are split over multiple routes.

Let $u$ be a source and destination node pair whose traffic demand is the largest among those in $\Psi$, where $u \in \Psi$. Initially, set $r$ to infinity.

- Step 1: Solve the corresponding LP problem and obtain the optimal solution for $x_{i j}^{p q}$ and $r$ by assuming $x_{i j}^{p q}$ is a real number with $0 \leq x_{i j}^{p q} \leq 1$. If no feasible solution exists, the algorithm stops.

- Step 2: Using $x_{i j}^{p q}$ obtained at Step 1, each sourcedestination pair $(p, q)$ is classified into either $\Phi$ or $\Psi$.

- Step 3: $(p, q)$ whose value of traffic demand is the largest in $\Psi$, is set in $u$. Set $m_{p q}=1$ as an initial value.

- Step 4: Out of neighbor links from the source node in $u$, except for the link to the ancestor node, find a set of $m_{p q}$ neighbor links that minimizes the congestion ratio after the traffic demand for $(p, q)$ is routed. If the obtained congestion ratio is smaller than $r$, replace $r$ with the obtained congestion and keep the set of neighbor links.

- Step 5: Increase $m_{p q}$ by one. If $m_{p q}$ does not exceed the maximum allowable split number, go to Step 4. Otherwise, go to Step 6.

- Step 6: If $r \leq 1.0,(p, q)$ in $u$ moves from $\Psi$ to $\Phi$. Otherwise, no feasible solution is found. The algorithm stops.

- Step 7: If $\Psi$ is empty, the solution has been obtained and The algorithm stops. Otherwise, go to Step 3.

\section{Performance evaluation}

The performance of the even-split S-OSPF scheme is compared to those of the split S-OSPF scheme, nonsplit S-OSPF scheme and the classical SPR scheme by solving LP problems. CPLEX [7] was used as the LP solver. The performance measure is the network congestion ratio, $r$. We used six sample networks to determine the basic characteristics of these schemes. All nodes in the network are assumed to behave as edge nodes. For the given network topologies, link capacities and traffic demands were randomly generated with uniform distribution in the range of $(8000,12000)$, and $(0,1000)$.

In even-split S-OSPF, non-split S-OSPF, split SOSPF, and classical SPR, the link weights that are used for shortest path computation are set to be inversely 


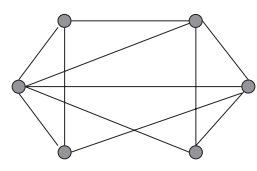

(a) Network 1

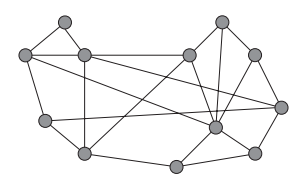

(c) Network 3

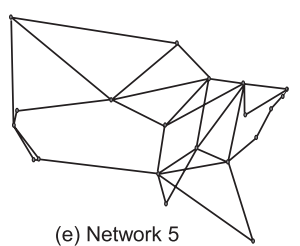

(e) Network 5
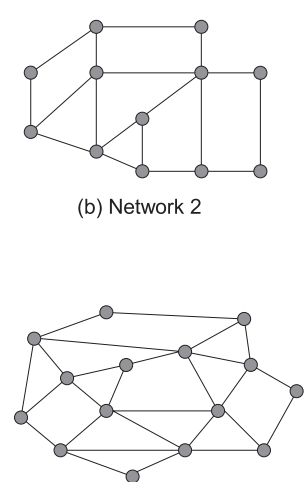

(d) Network 4

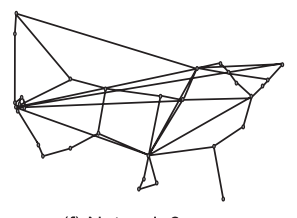

(f) Network 6 (b) Network 2

Fig. 5 Sample networks.

Table 5 Characteristics of networks.

\begin{tabular}{rrrr}
\hline Network type & No. of nodes & No. of links & Average node degree \\
\hline \hline Network 1 & 6 & 24 & 4.00 \\
Network 2 & 12 & 36 & 3.00 \\
Network 3 & 12 & 48 & 4.00 \\
Network 4 & 15 & 56 & 3.73 \\
Network 5 & 20 & 68 & 3.40 \\
Network 6 & 35 & 100 & 2.86 \\
\hline
\end{tabular}

proportional to the link capacities. The network congestion ratios are compared by using the sample networks, as shown in Figure 5. Networks 1-4 are presented in [2]. Networks 5 and 6 are presented in [4]. Networks 1-4 in Figure 1(a)-(d) are used as typical backbone networks [2]. Networks 5 and 6 in Figure 1 (e)-(f) are presented in [4], which are the Cable and Wireless network and CRL network, respectively, available at [8].

The characteristics of the networks are shown in Table 5. We obtained the average values of the normalized network congestion ratios for 100 randomly generated.

Figure 6 shows the result of congestion ratios of each scheme. To compare the network congestion ratios of the different schemes, we normalize those of even-split, non-split S-OSPF and split S-OSPF by that of classical SPR.

Even-split S-OSPF reduces the congestion ratio by up to $15 \%$ for all examined networks, compared to nonsplit S-OSPF. As network size increases, the difference

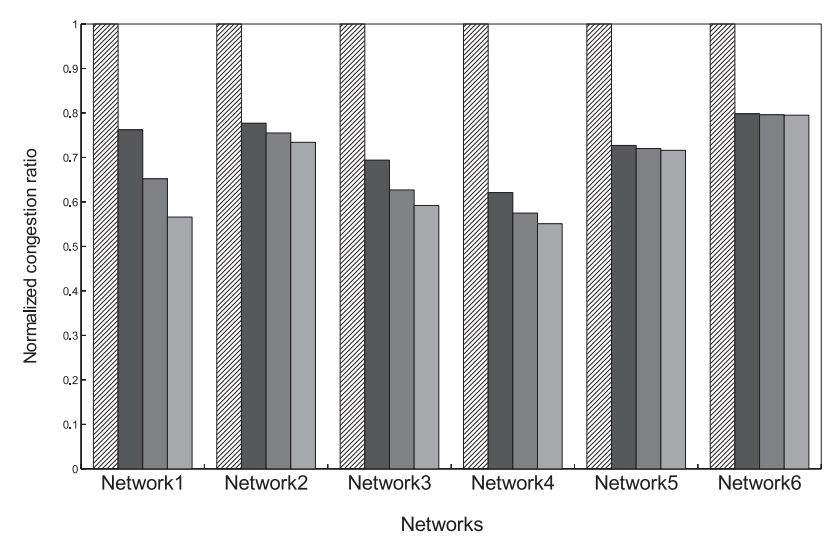

$\square$ Classical SPR $\square$ Non-split S-OSPF $\square$ Even-split S-OSPF $\square$ Split S-OSPF

Fig. 6 Congestion ratios in sample networks.

Table 6 Computation time in second in sample networks.

\begin{tabular}{rrrr}
\hline Network type & split [s] & non-split [s] & even-split [s] \\
\hline \hline Network 1 & 0.009992 & 0.010664 & 0.125031 \\
Network 2 & 0.045688 & 0.048170 & 0.181556 \\
Network 3 & 0.067903 & 0.065895 & 0.527589 \\
Network 4 & 0.133145 & 0.184871 & 0.683707 \\
Network 5 & 0.257007 & 0.263198 & 0.964206 \\
Network 6 & 2.862713 & 2.952775 & 3.096194 \\
\hline
\end{tabular}

in network congestion shrinks. In network 6 , the difference is about $1 \%$. This indicates that even-split S-OSPF is effectively matched the load-balancing performance of split-S-OSPF for large networks.

Table 6 compares with the computation time those of proposed scheme in sample networks. The difference of the computation times between split S-OSPF and even-split S-OSPF is caused by steps 2-7 of the heuristic algorithm in even-split S-OSPF. Note that, since the difference is lower than one second, it is not a strong impact from a practical point of view.

In Addition, the quantitative performance of the even-split S-OSPF is evaluated in several general networks with respect to three network parameters: the number of nodes, the number of adjacency nodes, and the average distance between nodes.

These networks are generated by using the BRITE [9] internet topology generator. BRITE generates a network according to the number of nodes $N$, the number of adjacency nodes $m$, and generation model for random topologies. The Waxman's model, used for interconnecting nodes of the topology, is given by ,

$$
P(u, v)=\alpha \exp \left(-\frac{d}{\beta L}\right),
$$

where $0 \leq \alpha, \beta \leq 1, d$ is the Eucidean distance from a 
Table 7 Characteristics of networks.

\begin{tabular}{|c|c|c|c|c|}
\hline$N$ & $\beta$ & $m$ & Number of links & Node degree \\
\hline 10 & 0.2 & 2 & 40 & 4.0 \\
\hline 10 & 0.2 & 3 & 54 & 5.4 \\
\hline 10 & 0.4 & 2 & 40 & 4.0 \\
\hline 10 & 0.4 & 3 & 54 & 5.4 \\
\hline 10 & 0.6 & 2 & 40 & 4.0 \\
\hline 10 & 0.6 & 3 & 54 & 5.4 \\
\hline 15 & 0.2 & 2 & 60 & 4.0 \\
\hline 15 & 0.2 & 3 & 90 & 6.0 \\
\hline 15 & 0.4 & 2 & 60 & 4.0 \\
\hline 15 & 0.4 & 3 & 90 & 6.0 \\
\hline 15 & 0.6 & 2 & 60 & 4.0 \\
\hline 15 & 0.6 & 3 & 90 & 6.0 \\
\hline 20 & 0.2 & 2 & 80 & 4.0 \\
\hline 20 & 0.2 & 3 & 120 & 6.0 \\
\hline 20 & 0.4 & 2 & 80 & 4.0 \\
\hline 20 & 0.4 & 3 & 120 & 6.0 \\
\hline 20 & 0.6 & 2 & 80 & 4.0 \\
\hline 20 & 0.6 & 3 & 120 & 6.0 \\
\hline 25 & 0.2 & 2 & 100 & 4.0 \\
\hline 25 & 0.2 & 3 & 150 & 6.0 \\
\hline 25 & 0.4 & 2 & 100 & 4.0 \\
\hline 25 & 0.4 & 3 & 150 & 6.0 \\
\hline 25 & 0.6 & 2 & 100 & 4.0 \\
\hline 25 & 0.6 & 3 & 150 & 6.0 \\
\hline 30 & 0.2 & 2 & 120 & 4.0 \\
\hline 30 & 0.2 & 3 & 180 & 6.0 \\
\hline 30 & 0.4 & 2 & 120 & 4.0 \\
\hline 30 & 0.4 & 3 & 180 & 6.0 \\
\hline 30 & 0.6 & 2 & 120 & 4.0 \\
\hline 30 & 0.6 & 3 & 180 & 6.0 \\
\hline
\end{tabular}

node to another node, and $L$ is the maximum distance between any two nodes. In this paper, $\beta$ is used to adjust the average distance from a node to another node. If $\beta$ is large, the average distance will become large.

We set up $\alpha=0.2$, and change other parameters, which are $N=10,15,20,25,30, m=2,3$, and $\beta=$ $0.2,0.4,0.6$. Therefore, we make $30(=5 \times 2 \times 3)$ parameter combinations. The characteristics of general networks are shown in Table 7 . We obtained the average values of the normalized network congestion ratios for 100 randomly generated. To compare the network congestion between even-split S-OSPF and classical SPR in Figures 7 and 8, we normalize the network congestion ratio of even-split S-OSPF by that of classical SPR.

Figure 7 shows the effectiveness of the average distance from a node to another node using parameter $\beta$. Even-split S-OSPF performance does not change much when $\beta$ is changed. It is because, when the average

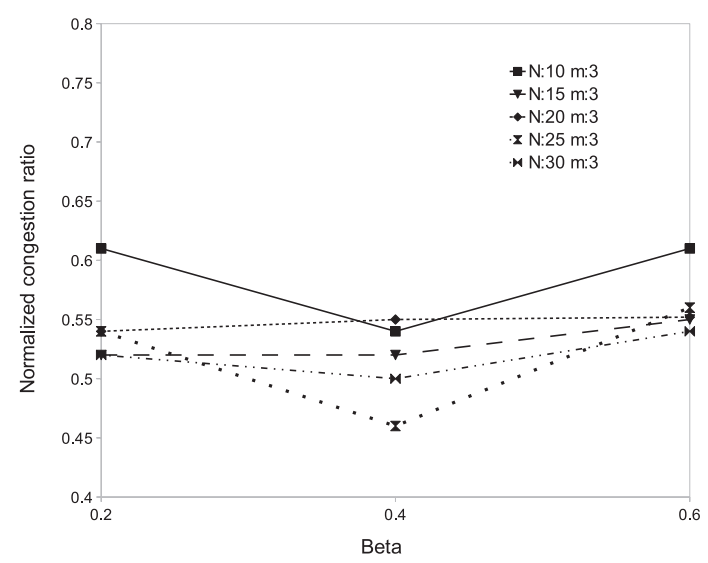

Fig. 7 Effectiveness of average distance.

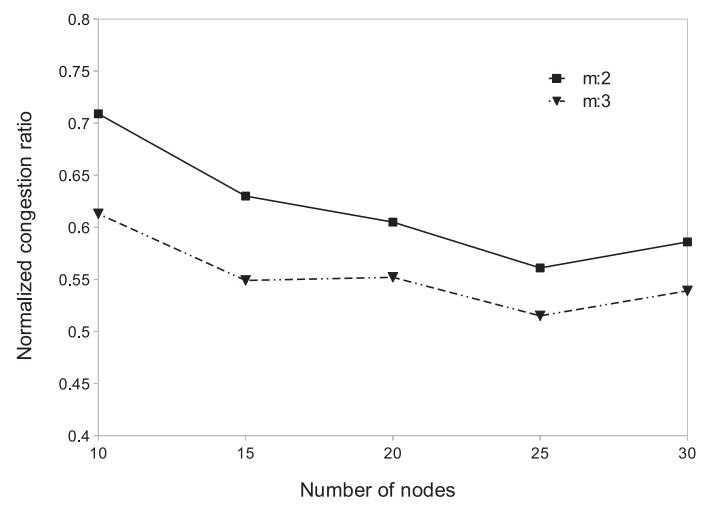

Fig. 8 Effectiveness of number of nodes and adjacency nodes.

distance increases, routes destined for near nodes become inefficient. Reversely, when the average distance decreases, routes destined far nodes require many used links. Therefore, the average distance does not affect the performance of even-split S-OSPF.

Figure 8 shows the dependency of achievable congestion ratio on the number of nodes $N$ and the number of adjacency nodes $m$ with fixed $\beta=0.6$. Even-split $\mathrm{S}-\mathrm{OSPF}$ reduces the congestion by up to $10 \%$ when the numbers of nodes and adjacency nodes become large. It is because, increasing the number of nodes causes a diversification of route. Moreover a higher number of adjacency nodes increase the freedom of route selection. Thus, even-split S-OSPF improves routing performance when the number of nodes and adjacency nodes are larger.

\section{Conclusions}

This paper proposed an even-split Smart-OSPF scheme to reduce network congestion ratio more than non-split S-OSPF, while kept the implementation of 
traffic distribution simpler than split S-OSPF. The routing decision problem was formulated as an ILP problem. We developed a heuristic algorithm, since it was difficult to solve the ILP problem in practical time. The performance of the heuristic algorithm was investigated in terms of network size. Numerical results showed that even-split S-OSPF offers better routing performance than non-split S-OSPF for small-size networks and matches that of split S-OSPF for large-size networks.

Moreover, we investigated the performance of evensplit S-OSPF to examine the dependency on network topologies. We focused attention on three network parameters, which are the number of nodes, the number of adjacency nodes, and average distance between two nodes. Numerical results via simulations showed that the average distance did not affect the performance of even-split S-OSPF, but the even-split S-OSPF improves routing performance when the number of nodes and adjacency nodes become large.

\section{References}

[1] Y. Wang and Z. Wang, "Explicit Routing Algorithms for Internet Traffic Engineering," IEEE International Conference on Conputer Communications and Networks (ICCCN), Oct. 1999.

[2] J. Chu and C. Lea, "Optimal Link Weights for Maximizing QoS Traffic," IEEE ICC 2007, pp.610-615, Jun. 2007.

[3] B. Fortz and M. Thorup, "Optimizing OSPF/IS-IS Weights in a Chnging World," IEEE Journal on Selected Areas in Communications, vol.20, no.4, pp.756-767, 2002.

[4] A.K. Mishra and A. Sahoo, "S-OSPF: a traffic engineeringsolution for OSPF based on best effrot networks," IEEE Globecom 2007, pp.1845-1849, Nov. 2007.

[5] E. Oki and A. Iwaki, "Load-Balanced IP Routing Scheme Basede on Shortest Paths in Hose Model," IEEE Trans. Commun., vol.58, no.7, pp.2088-2096, 2009.

[6] S. Tsunoda, A.H.A. Muktadir, and E. Oki, "LoadBalanced Shortest-Path-Based Routing Without Traffic Splitting in Hose Model,” IEEE ICC, Jun. 2011.

[7] http://www.ilog.com/, 2012.

[8] "Mapnet," online, http://www.caida.org/tools/visualization/mapnet/Backbones/, 2012.

[9] http://www.csbu.edu/brite/, 2012.

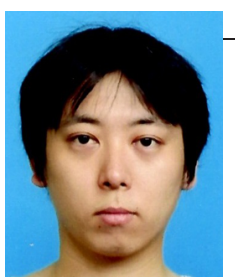

Masashi HONMA

Masashi HONMA received the B.E. in Information and Communications Engineering from The University of Electro-Communications, Tokyo Japan, in 2011. He is with Graduate School of Informatics and Engineering, The University of Electro-Communications. His research interests include routing and traffic control in IP networks.

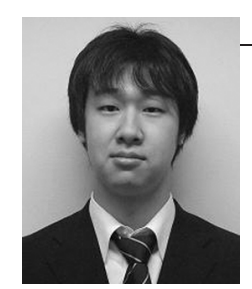

\section{Shunichi TSUNODA}

Shunichi TSUNODA received the B.E. in Information and Communications Engineering and M.E. in Communication Engineering and Informatics from The University of Electro-Communications, Tokyo Japan, in 2010 and 2012, respectively. His research interests include routing and optimization in IP networks. 


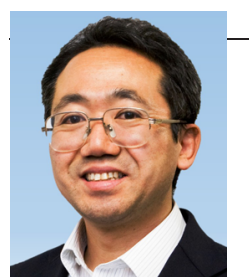

Eiji OKI

Eiji OKI is an Associate Professor at The University of ElectroCommunications, Tokyo, Japan. He received the B.E. and M.E. degrees in instrumentation engineering and a $\mathrm{Ph} . \mathrm{D}$. degree in electrical engineering from Keio University, Yokohama, Japan, in 1991, 1993, and 1999, respectively. In 1993, he joined Nippon Telegraph and Telephone Corporation (NTT) Communication Switching Laboratories, Tokyo, Japan. He has been researching network design and control, trafficcontrol methods, and high-speed switching systems. From 2000 to 2001, he was a Visiting Scholar at the Polytechnic Institute of New York University, Brooklyn, New York, where he was involved in designing terabit switch/router systems. He was engaged in researching and developing high-speed optical IP backbone networks with NTT Laboratories. He joined the University of Electro-Communications, Tokyo, Japan, in July 2008. He has been active in standardization of path computation element (PCE) and GMPLS in the IETF. He wrote more than ten IETF RFCs and drafts. Prof. Oki was the recipient of the 1998 Switching System Research Award and the 1999 Excellent Paper Award presented by IEICE, the 2001 Asia-Pacific Outstanding Young Researcher Award presented by IEEE Communications Society for his contribution to broadband network, ATM, and optical IP technologies, and the 2010 Telecom System Technology Prize by the Telecommunications Advanced Foundation. He has author/co-authored four books, Broadband Packet Switching Technologies, published by John Wiley, New York, in 2001, GMPLS Technologies, published by CRC Press, Boca Raton, FL, in 2005, Advanced Internet Protocols, Services, and Applications, published by Wiley, New York, in 2012, and Linear Programming and Algorithms for Communication Networks, CRC Press, Boca Raton, FL, in 2012. He is an IEEE Fellow and an IEICE Senior Member. 